\title{
Renormalization of Optical Transition Strengths in Semiconductor Nanoparticles due to Band Mixing
}

\author{
Kirill A. Velizhanin* \\ Theoretical Division, Los Alamos National Laboratory, Los Alamos, NM 87545
}

\begin{abstract}
Unique optical properties of semiconductor nanoparticles (SN) make them very promising in the multitude of applications including lasing, light emission and photovoltaics. In many of these applications it is imperative to understand the physics of interaction of electrons in a SN with external electromagnetic fields on the quantitative level. In particular, the strength of electron-photon coupling determines such important SN parameters as the radiative lifetime and absorption cross section. This strength is often assumed to be fully encoded by the so called Kane momentum matrix element. This parameter, however, pertains to a bulk semiconductor material and, as such, is not sensitive to the quantum confinement effects in SNs. In this work we demonstrate that the quantum confinement, via the so called band mixing, can result in a significant suppression of the strength of electron interaction with electromagnetic field. Within the envelope function formalism we show how this suppression can be described by introducing an effective energy-dependent Kane momentum. Then, the effect of band mixing on the efficiencies of various photoinduced processes can be fully captured by the conventional formulae (e.g., spontaneous emission rate), once the conventional Kane momentum is substituted with the renormalized energy-dependent Kane momentum introduced in here. As an example, we evaluate the energy-dependent Kane momentum for spherical PbSe and PbS SNs (i.e., quantum dots) and show that neglecting band mixing in these systems can result in the overestimation of absorption cross sections and emission rates by a factor of $\sim 2$.
\end{abstract}

Keywords: narrow-gap semiconductors, quantum dots, optical transitions, envelope function formalism, gauge invariance

\section{Introduction}

Unique properties of photoinduced electronic processes in semiconductor nanoparticles (SN), as compared to bulk semiconductors, is what makes SNs (e.g., quantum dots, nanorods) so promising in the multitude of applications including photovoltaics, photonics, lasing and light-emitting devices $[1,2,3,4,5,6,7]$. As follows from the term "photoinduced", such processes are all initiated by an optical absorption of a photon (or multiple photons) resulting in electronic transitions within a SN. The efficiency of initiation of such processes is thus directly determined by the strength of interaction between external electromagnetic fields (EMF) and SN electronic transitions. The same interaction does often further affect the electronic dynamics of initially photoexcited charge carriers. For example, the electron-EMF coupling determines the rate of Förster resonance energy transfer between nearby SNs. Photon absorption, as well as spontaneous and stimulated photon emissions, are main processes determining the efficiency of lasing. Finally, the lifetime of a charge carrier is affected by the electron-EMF coupling via the radiative recombination rate. This is especially true for high-quality SNs where the quantum yield of photoluminescence can approach $100 \%$,

\footnotetext{
${ }^{*}$ Corresponding author

Email address: kirill@lanl.gov (Kirill A. Velizhanin)
} 
implying that the observable lifetime of charge carriers approaches the inverse radiative recombination rate $[8,9]$.

The strength of coupling between electronic transitions in SNs and EMF is often expressed via the so called Kane momentum matrix element or simply Kane momentum, $P=\hbar\left\langle u_{c}|\nabla| u_{v}\right\rangle$, where $u_{c}$ and $u_{v}$ are bulk Bloch functions corresponding to conduction and valence band edges, respectively [10]. For example, approximating an SN as a simple two-level quantum emitter in vacuum results in the following expression for the rate of radiative recombination (in Gaussian units) [11, 12]

$$
k_{r}=\frac{4 e^{2} E P^{2}}{3 \hbar^{2} m^{2} c^{3}},
$$

where $e=|e|$ and $m$ are the magnitude of the charge and the mass of free electron, respectively. Speed of light in vacuum and Planck constant are denoted by $c$ and $\hbar$, respectively. Energy of the lowest electronic transition, $E$, could be significantly larger than the bandgap energy of the corresponding bulk semiconductor, $E_{g}$, due to quantum confinement effects in SNs. Cross sections of absorption and stimulated emission are linearly related to $k_{r}$ via Einstein coefficients [13], and so are also proportional to $P^{2}$. For simplicity, we disregard the effect of dielectric screening within the SN and the surrounding material (e.g., solvent) on the electron-EMF coupling. These effects can always be straightforwardly accounted for, as described in Ref. [14].

Representing a wavefunction of a conduction (valence) band electron in SN as a product of a slowly varying envelope function and the Bloch function $u_{c}\left(u_{v}\right)[10,15]$, one can relate the Kane momentum to the transition dipole moment of the lowest transition as $d=\left|\left\langle u_{c}|e \mathbf{r}| u_{v}\right\rangle\right|=\frac{e \hbar P}{m E_{g}}[16,15]$, as discussed in more detail in Sec. 6.2. Using this transition dipole moment, the radiative recombination rate can now be written as $[17]$

$$
k_{r}=\frac{4 E^{3} d^{2}}{3 \hbar^{4} c^{3}} .
$$

Since $P$ and $d$ are bulk properties, and as such are independent of specific value of transition energy $E$, Eqs. (1) and (2) imply $k_{r} \propto E$ in and $k_{r} \propto E^{3}$, respectively. Therefore, Eqs. (1) and (2) do contradict each other. This apparent contradiction in scaling of $k_{r}$ with respect to the energy of the lowest transition can often be ignored for wide-bandgap SNs (e.g., CdSe or CdS), where $E \approx E_{g}$ for typical experimentally relevant SN sizes. However, $E / E_{g}$ could be as high as $\sim 3-5$ in small narrow-gap SNs due to strong quantum confinement. Under these conditions, the issue of contradictory energy scaling in Eqs. (1) and (2) becomes critical and has to be resolved.

The main goal of the present paper is to carefully investigate the effect of quantum confinement in narrowgap SNs on the strength of coupling between electronic transitions and EMF. Working within the envelope function approximation $(k \cdot p$ model $)[10,15]$, we demonstrate that the requirement of gauge invariance of the $k \cdot p$ model disallows the energy independence of $P$ and $d$ in Eqs. (1) and (2). Specifically, it is demonstrated how the band mixing (or band coupling) $[18,19]$ in SNs renormalizes bulk values of $P$ and $d$ by making them effectively energy-dependent. Such renormalized energy-dependent parameters, $\tilde{P}(E)$ and $\tilde{d}(E)$, do yield exactly the same $k_{r}(E)$, once substituted into Eqs. (1) and (2) instead of bulk $P$ and $d$, respectively. This further implies that $E^{2} \tilde{d}^{2}(E)$ has to be linearly proportional to $\tilde{P}^{2}(E)$ as a function of $E$, and we show how this relation follows exactly from the gauge invariance of the $k \cdot p$ model. Using a simple one-dimensional two-band Kane model, we show how different regimes of quantum confinement affect the strength of the electron-EMF interaction. We further discuss a specific experimentally relevant example of $\mathrm{PbSe}$ and $\mathrm{PbS}$ spherical SNs, i.e., quantum dots (QD), and show that even though renormalized $\tilde{P}(E)$ and $\tilde{d}(E)$ are both energy-dependent, $\tilde{P}(E)$ dependence on energy is much weaker than that of $\tilde{d}(E)$, so the former could be represented by a simple linear fit (or even constant) within the experimentally relevant range of QD sizes. This result is consistent with previous experimental and theoretical observations [20, 21]. Fig. 1 plots the renormalized value of the Kane momentum for the lowest-energy optical transition in PbSe and PbS QDs, as evaluated within a suitable $k \cdot p$ model [22], as a function of the transition energy. As is seen, the square of the renormalized Kane momentum does change by $\sim 40 \%$ in the experimentally relevant energy window $(E=0.5-2 \mathrm{eV})$. This result can be directly used to evaluate radiative lifetimes in PbSe and PbS QDs. 


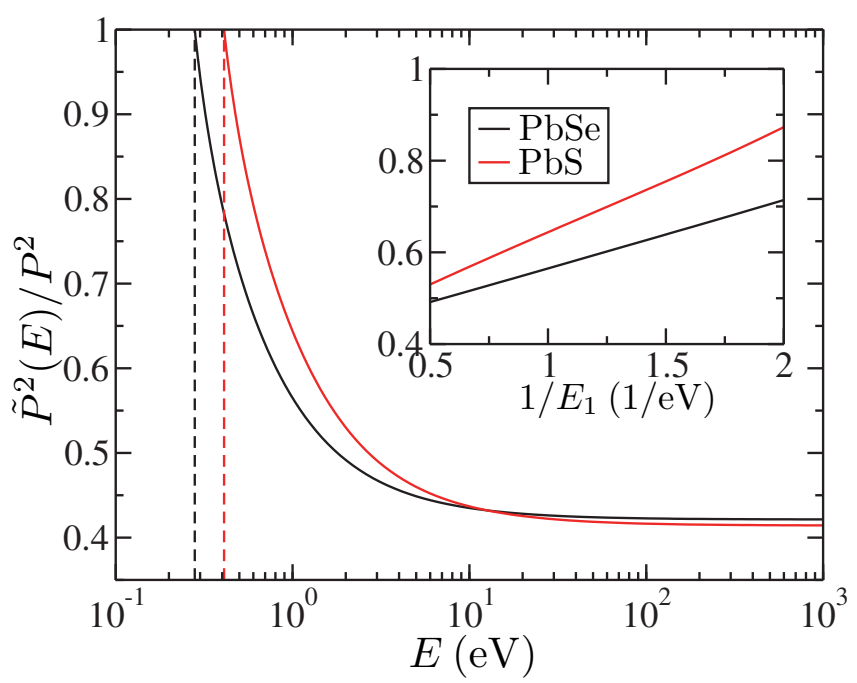

Figure 1: Normalized square of the effective energy-dependent Kane momentum for the lowest-energy transition in PbSe (black) and $\mathrm{PbS}$ (red) QDs as a function of transition energy, E. Vertical dashed lines represent bulk bandgap energies. Inset shows the same data within the experimentally relevant energy range.

The paper is organized as follows. The microscopic Hamiltonian for an electron in a bulk semiconductor in the presence of EMF is discussed in Sec. 2. Sec. 3 focuses on the envelope function Hamiltonian suitable for description of electronic structure and optical transitions in SNs. The effect of band mixing on the strength of optical transitions in a one-dimensional two-band Kane model is considered in Sec. 4. In particular, band mixing in the continuos and spatially confined Kane models is described in Secs. 4.1 and 4.2, respectively. Practically relevant example of lead chalcogenide QDs is discussed in Sec. 5. Sec. 6 reveals the necessity of accounting for band mixing when evaluating the intensities of optical transitions, and shows how disregarding band mixing often results in breaking the gauge invariance of the $k \cdot p$ model. Sec. 7 concludes.

\section{Microscopic Hamiltonian}

A single-particle Hamiltonian for an electron in a bulk semiconductor in the absence of external EMF reads as (vectors are written in bold)

$$
H_{0}(\mathbf{r}, \mathbf{p})=\frac{\mathbf{p}^{2}}{2 m}+V(\mathbf{r}, \mathbf{p})
$$

where $\mathbf{r}$ is the position vector, and $\mathbf{p}=-i \hbar \nabla$ is the momentum operator. The operator of potential energy, $V(\mathbf{r}, \mathbf{p})$, is periodic over the semiconductor lattice and can, in general, have contributions linear in momentum $\mathbf{p}$ due to spin-orbit interaction.

The direct evaluation of the radiative recombination rate from the microscopic Hamiltonian is somewhat tedious since it requires quantization of EMF. Optical absorption is simper to assess as the EMF can be treated classically. Therefore, in this paper we focus on the strength of coupling of electronic transitions to the classical EMF with the understanding that renormalized $\tilde{P}(E)$, obtained from such analysis, is exactly the same $\tilde{P}(E)$ that enters Eq. (1), by virtue of Einstein coefficients [13].

Interaction with a time-dependent classical EMF is introduced into Eq. (3) via the so called minimalcoupling procedure: (i) the momentum operator is "elongated" by the vector potential, $\mathbf{p} \rightarrow \mathbf{p}+\frac{e}{c} \mathbf{A}(\mathbf{x}, t)$, and (ii) the scalar potential $\varphi(\mathbf{r}, t)$ is added, so that the resulting Hamiltonian is (in Gaussian units) [23]

$$
H(\mathbf{r}, \mathbf{p}, t)=H_{0}\left(\mathbf{r}, \mathbf{p}+\frac{e}{c} \mathbf{A}(\mathbf{r}, t)\right)-e \varphi(\mathbf{r}, t) .
$$


The gauge transformation of the vector and scalar potentials is given by

$$
\begin{aligned}
& \mathbf{A}(\mathbf{r}, t) \rightarrow \tilde{\mathbf{A}}(\mathbf{r}, t)=\mathbf{A}(\mathbf{r}, t)+\nabla f(\mathbf{r}, t), \\
& \varphi(\mathbf{r}, t) \rightarrow \tilde{\varphi}(\mathbf{r}, t)=\varphi(\mathbf{r}, t)-\frac{1}{c} \frac{\partial f(\mathbf{r}, t)}{\partial t},
\end{aligned}
$$

where $f(\mathbf{r}, t)$ is an arbitrary real scalar function. It is straightforward to demonstrate that if an arbitrary wavefunction $\Psi(\mathbf{r}, t)$ is the solution of the time-dependent Schrödinger equation with Hamiltonian (4), then $\tilde{\Psi}(\mathbf{r}, t)=e^{-i \frac{e}{\hbar c} f(\mathbf{r}, t)} \Psi(\mathbf{r}, t)$ is the solution of the Schrödinger equation with the same Hamiltonian where the vector and scalar potentials are transformed according to Eq. (5). Therefore, the gauge transformation of the vector and scalar potentials is exactly equivalent to a certain unitary transformation of the wavefunction, which obviously does not affect any measurable quantities, e.g., charge density $|\Psi(\mathbf{x}, t)|^{2}$ or rates of photoinduced transitions between the states. The dynamics of the system, encoded by Hamiltonian (4), is thus gauge invariant. In what follows, we will loosely refer to Hamiltonians of the type given by Eq. (4) as gauge invariant, even though, strictly speaking, the Hamiltonian operator does not transform into itself upon substitution (5).

Although physical observables are gauge invariant, certain calculations could be simplified by the appropriate choice of gauge. Two specific gauge choices are of particular convenience when describing the interaction of SNs with EMF. Starting from an arbitrary gauge it is always possible to choose $f(\mathbf{r}, t)=$ $-c \int_{t_{0}}^{t} d t^{\prime} \varphi\left(\mathbf{r}, t^{\prime}\right)$ in Eq. (5) so that the scalar potential vanishes exactly upon the gauge transformation, resulting in

$$
\mathbf{A}(\mathbf{r}, t)=\mathbf{A}(t)=\mathbf{A}_{0} \sin \left(\omega_{0} t\right) ; \varphi(\mathbf{r}, t) \equiv 0,
$$

where it is assumed that the electronic transitions in SN are driven by the oscillatory EMF of angular frequency $\omega_{0}$. The constant vector amplitude of the EMF vector potential is $\mathbf{A}_{0}$. The dependence of the vector potential on $\mathbf{r}$ is neglected as it is typically very weak for, e.g., electromagnetic waves of relevant frequencies. For the free electromagnetic wave, the gauge (6) coincides with the Coulomb (transverse) gauge. If EMF is weak, we can expand the microscopic Hamiltonian (4) with respect to the vector potential and retain only up to linear terms. If we further neglect the spin-orbit interaction, the resulting electron-EMF coupling operator is proportional to $\mathbf{A}_{0} \cdot \mathbf{p}$. Since momentum $\mathbf{p}$ is related to velocity, gauge (6) is often called the velocity gauge.

The second convenient choice of gauge can be obtained from Eq. (6) by performing the gauge transformation, Eq. (5), with $f(\mathbf{r}, t)=-\left(\mathbf{r} \cdot \mathbf{A}_{0}\right) \sin \left(\omega_{0} t\right)$. This produces

$$
\mathbf{A}(\mathbf{r}, t) \equiv 0 ; \varphi(\mathbf{r}, t)=\frac{\omega_{0}}{c}\left(\mathbf{r} \cdot \mathbf{A}_{0}\right) \cos \left(\omega_{0} t\right)
$$

Substituting this expression into Eq. (4) one can see that the electron-EMF coupling operator is linearly proportional to $\mathbf{r}$. Hence, gauge (7) is often referred to as the length gauge.

The convenience of the two gauge choices, Eqs. (6) and (7), stems from the fact that electronic transitions in SNs are coupled to EMF solely through either vector or scalar potential for the velocity and length gauge, respectively. It has to be emphasized here, however, that we are able to make the vector potential vanish in Eq. (7) only because we neglected its coordinate dependence in Eq. (6). More generally, one can make a vector potential vanish only locally in space by choosing a gauge.

The gauge invariance is absolutely necessary for the accurate and consistent treatment of interaction of charge carriers with EMF. For example, neglecting the vector potential in Eq. (4), and thus breaking gauge invariance, can result in unphysical gauge-dependent rates of photoinduced transitions, which could be made as large as desired by a choice of gauge. An example of such behavior is provided in Sec 6.1.

\section{Envelope function approximation}

To analyze the electronic structure of SNs, it is convenient to not directly use Hamiltonian (3), but to transform it first to the envelope function representation $[10,15]$. To this end, we find the eigenfunctions 
$\chi_{n \mathbf{k}}(\mathbf{r})$ of the bulk Hamiltonian (3) at a specific (typically high symmetry) point $\mathbf{k}$ of the Brillouin zone

$$
\chi_{n \mathbf{k}}(\mathbf{r})=\frac{1}{\sqrt{V}} e^{i \mathbf{k} \cdot \mathbf{r}} u_{n \mathbf{k}}(\mathbf{r})
$$

Here, index $n$ enumerates electronic bands, and Bloch functions $u_{n \mathbf{k}}(\mathbf{r})$ are periodic over the lattice. These eigenfunctions can now be used to expand an arbitrary time-dependent wavefunction $\Psi(\mathbf{r}, t)$ as

$$
\Psi(\mathbf{r}, t)=\sum_{n} \phi_{n}(\mathbf{r}, t) \chi_{n \mathbf{k}}(\mathbf{r})
$$

where $\phi_{n}(\mathbf{r}, t)$ are the so called envelope functions that are assumed to vary slowly over the size of a single unit cell. Since the basis functions $\chi_{n \mathbf{k}}(\mathbf{r})$ are time-independent, it is possible to write down an effective low-energy Hamiltonian that acts only within a space of envelope functions. This is done by multiplying the Hamiltonian (3) by the basis set functions from both sides and then averaging over the unit cell (uc)

$$
H_{0, n m}(\mathbf{r}, \mathbf{p})=\left\langle\chi_{n \mathbf{k}}\left|H_{0}(\mathbf{r}, \mathbf{p})\right| \chi_{m \mathbf{k}}\right\rangle_{\mathbf{u c}} .
$$

Hamiltonian $H_{0, n m}$ is a matrix of operators that is at most quadratic with respect to p. Typically, such Hamiltonians include terms proportional to the dot product of $\mathbf{k}$ and $\mathbf{p}$, hence the term $k \cdot p$ Hamiltonian. These operators act only within the space of envelope functions, so that a time-dependent Schrödinger equation for the wavefunction in Eq. (9) can now be expressed as

$$
\sum_{m} H_{0, n m}(\mathbf{r}, \mathbf{p}) \phi_{m}(\mathbf{r}, t)=i \hbar \frac{\partial \phi_{n}(\mathbf{r}, t)}{\partial t} .
$$

The obtained Hamiltonian $H_{0, n m}(\mathbf{r}, \mathbf{p})$ is still not quite suitable for practical calculations since the summation over $m$ in Eq. (11) runs over an infinite number of electronic bands. The number of bands can be reduced to a finite number of relevant ones by means of an approximate canonical transformation that decouples the so called far-bands from the experimentally relevant ones (e.g., highest valence and lowest conduction bands) within the second-order perturbation theory $[24,19]$. The resulting Schrödinger equation is symbolically the same as the one in Eq. (11), but the summation is now restricted to few bands. The canonical transformation, typically restricted to the second-order perturbation theory, does not introduce any higher powers of the momentum operator, so the components of resulting few-band matrix Hamiltonian are still at most quadratic with respect to $\mathbf{p}$.

Within the envelope function formalism, the interaction of EMF with charge carriers could be dealt with using two different approaches. The first approach is to use the envelope functions, obtained from Eq. (11), to restore the entire microscopic wavefunction (9). Coupling between such wavefunctions due to EMF could then be evaluated using the microscopic Hamiltonian (4). The problem with this approach is that the canonical transformation, used to decouple far-bands, also transforms the basis (8), so that the new basis functions are linear combinations of the old ones with, in general, time- and coordinate-dependent coefficients. Under these conditions, the restoration of the microscopic wavefunction is a tedious task that has to be performed very accurately to preserve the gauge invariance of the problem.

The other, perhaps more physically transparent approach, is to include the interaction with the vector and scalar potentials directly into the envelope function Hamiltonian, Eq. (10). This can always be done directly by starting from the microscopic Hamiltonian (4), and then averaging it over the unit cell and performing the canonical transformation to reduce the number of bands [24]. The result, however, can be guessed because the final result $-k \cdot p$ Hamiltonian with external fields - must be gauge-invariant in the sense introduced in Sec. 2. More specifically, since the gauge transformation of the scalar and vector potentials is identical to unitary transformation of a time-dependent wave function, one can conclude that the external EMF has to be introduced into $H_{0, n m}(\mathbf{r}, \mathbf{p})$ as

$$
\begin{gathered}
H_{0, n m}(\mathbf{x}, \mathbf{p}) \rightarrow H_{n m}(\mathbf{x}, \mathbf{p}, t) \\
=H_{0, n m}\left(\mathbf{x}, \mathbf{p}+\frac{e}{c} \mathbf{A}(\mathbf{x}, t)\right)-e \delta_{n m} \varphi(\mathbf{x}, t) .
\end{gathered}
$$


The result is of course very similar to how it was done in the case of the microscopic Hamiltonian, Eq. (4), since the minimal-coupling procedure is a natural gauge-invariant way to introduce vector and scalar potentials into a generic Hamiltonian. We wish to emphasize here that once the EMF is introduced into $H_{0, n m}$, one does not need to restore the microscopic wavefunctions to perform calculations in the presence of EMF, since the dynamics of charge carriers in the presence of EMF is fully encoded by the just obtained envelope function Hamiltonian (12). For example, rates of photoinduced transitions between electronic states could be obtained by treating terms containing $\mathbf{A}(\mathbf{r}, t)$ and $\varphi(\mathbf{r}, t)$ in $H_{n m}(\mathbf{r}, \mathbf{p}, t)$ as a perturbation. More specifically, since components of Hamiltonian $H_{0, n m}$ are at most quadratic with respect to $\mathbf{p}$, the lowest order operator of interaction with EMF can be obtained from Eq. (12) as

$$
\begin{gathered}
F_{n m}(\mathbf{r}, \mathbf{p}, t)=\frac{e}{2 c}\left[\frac{\partial H_{0, n m}(\mathbf{r}, \mathbf{p})}{\partial \mathbf{p}} \cdot \mathbf{A}(\mathbf{r}, t)\right. \\
\left.+\mathbf{A}(\mathbf{r}, t) \cdot \frac{\partial H_{0, n m}(\mathbf{r}, \mathbf{p})}{\partial \mathbf{p}}\right]-e \delta_{n m} \varphi(\mathbf{r}, t) .
\end{gathered}
$$

For specific gauge choices, Eqs. (6) and (7), this operator becomes

$$
F_{1, n m}(\mathbf{r}, \mathbf{p}, t)=\frac{e}{c}\left[\mathbf{A}_{0} \cdot \frac{\partial H_{0, n m}(\mathbf{r}, \mathbf{p})}{\partial \mathbf{p}}\right] \sin \left(\omega_{0} t\right),
$$

and

$$
F_{2, n m}(\mathbf{r}, \mathbf{p}, t)=-\delta_{n m} \frac{e \omega_{0}}{c}\left(\mathbf{r} \cdot \mathbf{A}_{0}\right) \cos \left(\omega_{0} t\right) .
$$

It is a tedious task to directly prove that operators $F_{1}$ and $F_{2}$, that are first-order in EMF, result in identical photoinduced transition rates [25]. However, since the Hamiltonian is gauge invariant, all physical observables are exactly gauge independent at EMF of arbitrary strength, and, therefore, for each order of field strength independently. The leading order terms of expansion of time-resolved state populations with respect to the EMF strength do thus produce gauge invariant transition rates.

\section{Two-band Kane model}

In this section we will explore the general features of how mixing between electronic bands affects the strength of the electron-EMF interaction. To this end, we will assume a simplest possible model with band mixing - a one-dimensional (1D) two-band Kane model, described by a Hamiltonian [26, 27, 28]

$$
H_{0}=\left(\frac{E_{g}}{2}+\frac{p^{2}}{2 m_{*}}\right) \sigma_{z}+\frac{P}{m} p \sigma_{x},
$$

where $p=-i \hbar \frac{\partial}{\partial x}$ and the Pauli matrices are

$$
\sigma_{x}=\left[\begin{array}{ll}
0 & 1 \\
1 & 0
\end{array}\right], \sigma_{y}=\left[\begin{array}{cc}
0 & -i \\
i & 0
\end{array}\right], \sigma_{z}=\left[\begin{array}{cc}
1 & 0 \\
0 & -1
\end{array}\right]
$$

In Eq. (16), $E_{g}$ is the bulk bandgap energy, $m_{*}$ is the effective mass of carriers (assumed identical for electrons and holes), and $P$ is the Kane momentum. The free electron mass is denoted by $m$. Rescaling length and energy results in a dimensionless Hamiltonian

$$
H_{0}=\left(1+p^{2}\right) \sigma_{z}+\alpha p \sigma_{x}
$$

where $\alpha$ is the dimensionless Kane momentum and $p=-i \frac{\partial}{\partial x}$. The operators of the lowest-order electronEMF interaction, Eqs. (14) and (15), become, respectively

$$
F_{1}(x, p, t)=A_{0}\left[2 p \sigma_{z}+\alpha \sigma_{x}\right] \sin \left(\omega_{0} t\right),
$$

and

$$
F_{2}(x, p, t)=-A_{0} x \omega_{0} I_{2} \cos \left(\omega_{0} t\right),
$$

where $I_{2}$ is the $2 \times 2$ unit matrix. These expressions were also made dimensionless by rescaling $A_{0}$. 


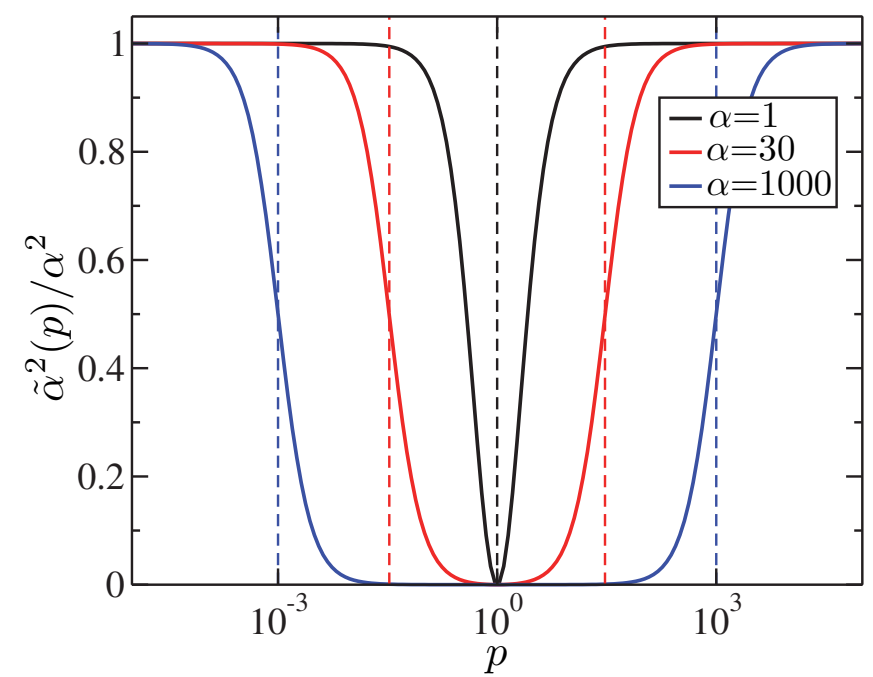

Figure 2: Square of the normalized effective Kane momentum as a function of $p$ for three different values of $\alpha=1$, 30 and 1000. The vertical dashed lines correspond to $p=\alpha^{-1}$ and $p=\alpha$ for each specific value of $\alpha$.

\subsection{Continuous 1D Kane model}

We first consider the effect of band mixing on the strength of electron-EMF coupling in the continuous Kane model, i.e., coordinate $x$ is unrestricted. In this case, the length gauge, Eq. (20), does result in illdefined transition amplitudes since scalar potential grows linearly with $x$. We, therefore, choose the velocity gauge, Eq. (19). The amplitude of photoinduced transitions between the eigenstates of Hamiltonian (18) is then encoded by operator

$$
f_{1}=2 p \sigma_{z}+\alpha \sigma_{x}
$$

so that Eq. (19) becomes $F_{1}=A_{0} f_{1} \sin \left(\omega_{0} t\right)$. Since the momentum $p$ becomes a parameter in the continuous Kane model, energies of eigenstates of Hamiltonian (18) are functions of this parameter

$$
E_{ \pm}(p)= \pm \sqrt{\left(1+p^{2}\right)^{2}+\alpha^{2} p^{2}}
$$

and the corresponding wavefunctions (bi-spinors) are

$$
\begin{aligned}
\Psi_{ \pm}(p)= & {\left[\alpha^{2} p^{2}+\left(E_{ \pm}(p)-1-p^{2}\right)^{2}\right]^{-1 / 2} } \\
& \times\left[\begin{array}{c}
\alpha p \\
E_{ \pm}(p)-1-p^{2}
\end{array}\right]
\end{aligned}
$$

The effective Kane momentum can now be defined via a transition intensity as

$$
\tilde{\alpha}^{2}(p)=\left|\left\langle\Psi_{+}(p)\left|f_{1}\right| \Psi_{-}(p)\right\rangle\right|^{2}=\frac{\alpha^{2}\left(1-p^{2}\right)^{2}}{\left(1+p^{2}\right)^{2}+\alpha^{2} p^{2}} .
$$

Fig. 2 shows the square of the normalized effective Kane momentum as a function of momentum $p$ for three different values of Kane momentum: $\alpha=1$ (sold black line), $\alpha=30$ (solid red line) and $\alpha=1000$ (solid blue line). As a function of $p, \tilde{\alpha}^{2}(p)$ is seen to have three distinct regimes: (i) plateau $\tilde{\alpha}^{2} / \alpha^{2}=1$ at very small momenta, (ii) plateau $\tilde{\alpha} \approx 0$ at intermediate values of $p$, and (iii) $\tilde{\alpha}^{2} / \alpha=1$ at very large momenta. These regimes are especially pronounced at $\alpha \gg 1$.

If momentum $p$ is not too large, i.e., in the first two regimes, the Hamiltonian (18) and the electron-EMF coupling (21) can be approximated as $H_{0}=\sigma_{z}+\alpha p \sigma_{x}$ and $f_{1}=\alpha \sigma_{x}$, respectively. This approximation is valid at $p \ll \alpha$. Since $f_{1}$ is proportional to $\sigma_{x}$, it most efficiently couples spinors that are eigenfunctions 
of $\sigma_{z}$. Since $H_{0} \approx \sigma_{z}$ at $p \ll \alpha^{-1}$, the electron-EMF coupling is most efficient is this regime, resulting in $\left|\left\langle\Psi_{+}\left|f_{1}\right| \Psi_{-}\right\rangle\right|=\alpha$. We thus associate condition $p \ll \alpha^{-1}$ with the first regime in Fig. 2, where the Kane momentum is not renormalized, i.e., $\tilde{\alpha}^{2}=\left|\left\langle\Psi_{+}\left|f_{1}\right| \Psi_{-}\right\rangle\right|^{2}=\alpha^{2}$. On the other hand, $H_{0} \approx \alpha p \sigma_{x}$ at $\alpha^{-1} \ll p \ll \alpha$, so that the eigenstates of $H_{0}$ are not coupled by $f_{1} \propto \sigma_{x}$ in this regime, resulting in $\tilde{\alpha}=0$. We thus associate condition $\alpha^{-1} \ll p \ll \alpha$ with the second regime in Fig. 2. Electron-EMF coupling is heavily suppressed in this regime resulting in $\tilde{\alpha}^{2} / \alpha^{2} \approx 0$. In the both first and second regimes, accounting for neglected terms in $H_{0}$ and $f_{1}$, e.g., the first term in Eq. (21), results in negligible corrections to already obtained values of $\tilde{\alpha}$.

At first glance, the third regime, $p \gg \alpha$, has to be very similar to the first one. Indeed, Hamiltonian (18) becomes diagonally dominated again, $H_{0} \approx p^{2} \sigma_{z}$, so one might follow the considerations above to conclude that $\tilde{\alpha}^{2} / \alpha^{2}=1$ in this regime. This result is indeed correct, although it turns out that the logic used when discussing the first regime is not quite applicable here. More specifically, the second term in Eq. (21) dominated the transition intensity in the first regime. In the third regime, however, the two terms of $f_{1}$ produce contributions to the transition intensity that are of the same order of magnitude. Actually, the contributions from $2 p \sigma_{z}$ and $\alpha \sigma_{x}$ to the matrix element in Eq. (24) are of the opposite signs, and the contribution of the former term is twice as large in magnitude as the contribution of latter one. Therefore, the value of matrix element in Eq. (24) changes from +1 to -1 when switching from the first regime to the third one. The third regime is thus physically rather distinct from the first one, although the resulting transition intensity is the same since Eq. (24) is insensitive to the phase of the transition matrix element.

With regards to the third regime, we would like to note here that even though this regime, $p \gg \alpha$, naturally appears in Hamiltonian (16), we expect it to be of less practical importance than the first two regimes, at least when treated within the envelope function approach. Indeed, the Kane momentum $P=$ $\hbar\left\langle u_{c}|\nabla| u_{v}\right\rangle$ can be thought of as a certain characteristic momentum associated with a single unit cell. Then, the condition $p \gg \alpha$ can only be realized when the envelope wavefunction is spatially confined to sizes not much larger than that of a single unit cell. At these conditions, the envelope function formalism breaks down. As an example, one can show that for the third regime to be realized in Eq. (16) in case of PbSe or $\mathrm{PbS}$ quantum dots [22], one has to have the quantum confinement energies significantly exceeding $1 \mathrm{eV}$. As is clearly seen from the bulk dispersion relations $[29,30]$, the standard $k \cdot p$ approximation for these materials becomes inaccurate at such energies and higher-energy bands have to be explicitly added into consideration.

\subsection{Spatially confined 1D Kane model}

In this subsection we analyze the effect of band mixing on the renormalization of the strength of electronEMF interaction in the presence of quantum confinement. To this end, we again adopt the two-band 1D Kane model, Eq. (18). To introduce the size quantization, a wavefunction is required to vanish at $x= \pm L / 2$, where $L$ is the quantum confinement length. Quantum-confined levels are then found as follows. First, all the continuous solutions for Hamiltonian (18) are found for a given energy. Since the resulting characteristic equation is of the forth order with respect to momentum $p$, four linearly independent continuous solutions, each being a bi-spinor, are obtained. Second, a linear combination of these solutions is required to vanish at $x= \pm L / 2$, which yields a homogeneous system of linear equations with four unknowns. The resulting secular equation yields the discrete energy spectrum, corresponding to the levels of the quantum-confined two-band 1D Kane model. This procedure is described in more detail in Appendix A.

The dependence of the energy of the lowest conduction band state on effective momentum $\tilde{p}_{1}=\pi / L$ is shown in Fig. 3. If the band mixing is absent (i.e., $\alpha=0$ ) or could be neglected, then Hamiltonian (18) encodes two uncoupled bands, each having simple parabolic dependence of energy on momentum. At these conditions, the problem of size quantization reduces to the textbook "particle in a box" problem with the result $E_{ \pm n}= \pm\left(1+\tilde{p}_{n}^{2}\right)$, where $n=1,2,3, \ldots$ and $\tilde{p}_{n}=\pi n / L$. Hamiltonian of the continuous Kane model (18) is diagonally dominated at either $p \ll \alpha^{-1}$ or $p \gg \alpha$, so, expectedly, the exact result for $E_{1}$ (thin red line) is well described by $E_{1}=\left(1+\tilde{p}_{1}^{2}\right)$ at very small or very large effective momenta.

The just obtained approximate expression for the energy at very large or very small momenta could be obtained from Eq. (22) by simply substituting $p \rightarrow \tilde{p}_{1}$ and then assuming small $\alpha$ limit. Analogously, one would expect that Eq. (22) yields $E_{1} \approx \alpha \tilde{p}_{1}$ in the intermediate confinement regime, $\alpha^{-1} \ll \tilde{p}_{1} \ll \alpha$. 


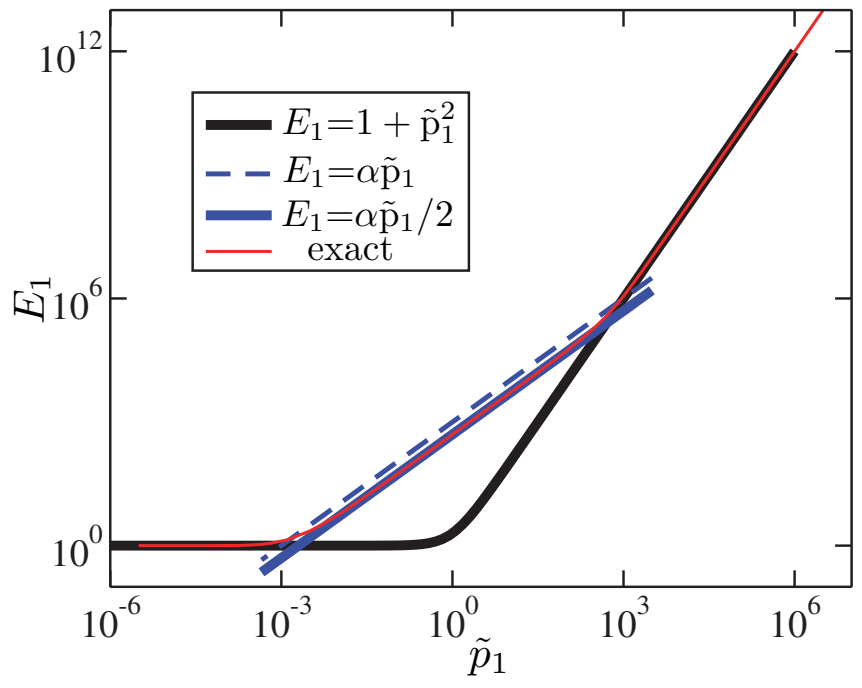

Figure 3: Energy of the lowest positive-energy state in a spatially confined Kane model $(\alpha=1000)$, plotted as a function of the effective momentum, $\tilde{p}_{1}=\pi / L$. Thin red line is the exact numerical result. Thick black line is the expected asymptotic behavior in the the regimes of the weak and strong confinement, $E_{1}=1+\tilde{p}_{1}^{2}$. Dashed blue lines represents the "naive" spatial quantization in the intermediate regime, $E_{1}=\alpha \tilde{p}_{1}$. Thick blue lines shows the correct asymptotic dependence in the intermediate regime, $E_{1}=\alpha \tilde{p}_{1} / 2$.

However, this dependence, shown by dashed blue line in Fig. (3) clearly deviates from the exact result (red line) by a constant prefactor. That the behavior of the system in this regime is more involved can already be suspected from the following consideration. In the intermediate regime, Hamiltonian (18) could be approximated as $H_{0} \approx \alpha p \sigma_{x}$. However, the quantum-confined states for such a Hamiltonian are not welldefined because this Hamiltonian, being only first-order with respect to momentum, yields only two linearly independent continuous solutions for each energy, and so the boundary conditions can not be satisfied. Therefore, the diagonal terms of the Hamiltonian (18) cannot be simple dropped even if $\alpha^{-1} \ll \tilde{p}_{1} \ll \alpha$. This leads to a suspicion that one cannot just take energies $E(p)= \pm \alpha p$ corresponding to continuous solutions of $H_{0} \approx \alpha p \sigma_{x}$, and then "quantum-confine" them by setting $p=\tilde{p}_{n}$. An accurate analysis of the effect of quantum confinement in this regime, provided in Appendix $\mathrm{A}$, results in $E_{1}=\alpha \tilde{p}_{1} / 2$. This result is plotted by a solid blue line in Fig. 3, demonstrating an excellent agreement with the exact numerical result (thin red line).

Fig. 4(a) shows the normalized transition intensity for the lowest energy transition, $E_{-1} \rightarrow E_{1}$, as a function of the effective momentum $\tilde{p}_{1}$. In the most illustrative case, $\alpha \gg 1$, one can again see three distinct regimes corresponding to those already observed in Fig. 3. The general trend of suppressing transition intensity when going from the weak $\left(\tilde{p}_{1} \ll \alpha^{-1}\right)$ to intermediate $\left(\alpha^{-1} \ll \tilde{p}_{1} \ll \alpha\right)$ quantum confinement is qualitatively similar to that observed for the continuous Kane model in Sec. 4.1. Quantitatively, however, the continuous and spatially confined Kane models are different in the intermediate regime. As discussed above, even though Hamiltonian (18) is off-diagonally dominated in this regime, the diagonal terms cannot be completely disregarded in the spatially confined case. Accounting for these terms results in only a partial suppression of the transition intensity in the intermediate regime. More quantitatively, the effective Kane momentum becomes $\tilde{\alpha}^{2} / \alpha=4 / \pi^{2}$ in the limit $\alpha \gg 1$, as derived in Appendix A. This asymptotic result, plotted by a horizontal dashed red line in Fig. 4(a), is seen to be in agreement with the exact dependence of $\tilde{\alpha}^{2} / \alpha^{2}$ on $\tilde{p}_{1}$ (blue line) in the intermediate confinement regime.

The transition intensity for the lowest transition drops even further when switching from the intermediate to the strong $\left(\tilde{p}_{1} \gg \alpha\right)$ confinement regime. Asymptotically, $\tilde{\alpha}^{2} / \alpha^{2}=[4 \operatorname{coth}(\pi / 2)-\pi]^{2} / \pi^{2} \approx 0.15$ in this regime when $\alpha \gg 1$, as derived in Appendix A and shown as a horizontal dashed black line in Fig. 4(a). This is rather different from $\tilde{\alpha}^{2} / \alpha^{2}=1$ obtained for the continuous Kane model. The discrepancy can ultimately be traced back to the fact that the band-mixing term in Eq. (18), i.e., $\alpha p \sigma_{x}$, does of course 


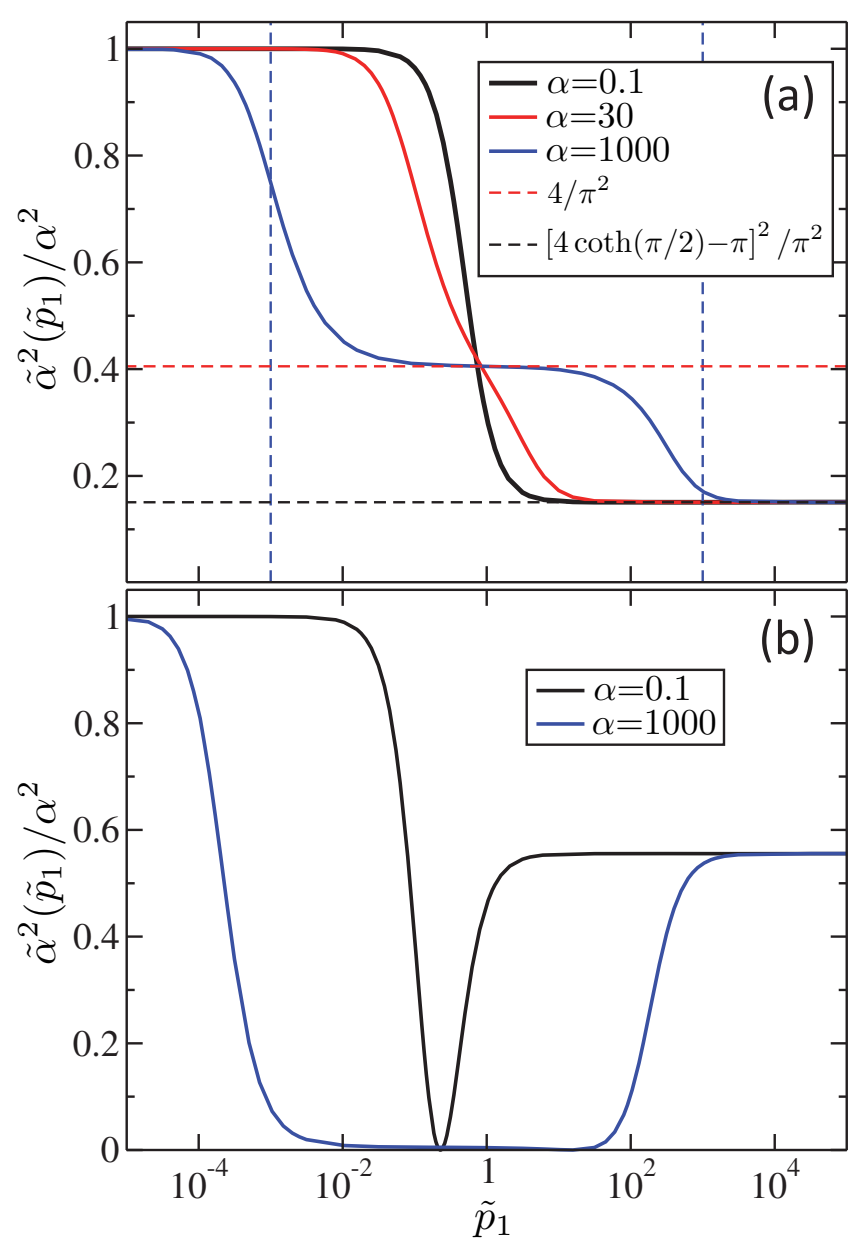

Figure 4: Panel (a) shows the normalized transition intensity for the lowest-energy transition, $E_{-1} \rightarrow E_{1}$, as a function of $\tilde{p}_{1}=\pi / L$ for three different values of Kane momentum: $\alpha=0.1,10$ and 1000. The vertical dashed lines correspond to $\tilde{p}_{1}=\alpha^{-1}$ and $\tilde{p}_{1}=\alpha$ for $\alpha=1000$. Panel (b) is the same except that it is not for the lowest transition, but for the $E_{-5} \rightarrow E_{5}$ transition. 
conserve momentum in the continuous case, but can couple states of different effective momenta $\tilde{p}_{n}$ in the spatially confined case. This latter effect has to be less important for higher-energy transitions where the effective momentum becomes more and more "conserved". One, therefore, can expect that the difference between continuous and spatially-confined Kane models with regards to the renormalization of transition intensity will become less pronounced at large transition energies. Fig. 4(b) shows the normalized intensity for the $E_{-5} \rightarrow E_{5}$ transition, and this is clearly seen that the dependence of $\tilde{\alpha}^{2} / \alpha^{2}$ on $\tilde{p}_{1}$ becomes very reminiscent to the results obtained previously for the continuous Kane model, Fig. 2. The spatially confined and continuous Kane models can therefore yield similar renormalization of the transition intensities at large transition energies. The lowest transition, however, experiences the most effect of the quantum confinement, resulting in a renormalization that is quite different for the two models considered in this section.

\section{Lead chalcogenide quantum dots}

In this section we consider a more realistic and experimentally relevant example - the effect of band mixing on the strength of the electron-EMF coupling in lead selenide (PbSe) and lead sulfide (PbS) spherically symmetric SNs (i.e., quantum dots). A four-band $k \cdot p$ Hamiltonian to describe electronic excitations in bulk lead chalcogenide (PbSe, PbS and PbTe) was originally introduced by Dimmock [31, 32], and Mitchell and Wallis [33]. The spherically symmetric version of this Hamiltonian reads as

$$
H_{0}=\left[\begin{array}{cc}
\left(\frac{E_{g}}{2}+\frac{p^{2}}{2 m_{c}}\right) I_{2} & \frac{P}{m}(\boldsymbol{\sigma} \cdot \mathbf{p}) \\
\frac{P}{m}(\boldsymbol{\sigma} \cdot \mathbf{p}) & -\left(\frac{E_{g}}{2}+\frac{p^{2}}{2 m_{v}}\right) I_{2}
\end{array}\right],
$$

where $m_{c}$ and $m_{v}$ are far-band contributions to the electron and hole effective masses, respectively. Bulk bandgap energy is denoted by $E_{g}$. Momentum operator is $\mathbf{p}=-i \hbar \nabla$. Kane momentum is denoted by $P$. Pauli vector is defined as $\boldsymbol{\sigma}=\left[\sigma_{x}, \sigma_{y}, \sigma_{z}\right]$, and $I_{2}$, as defined before, is the $2 \times 2$ unit matrix. Energies, $E_{n}$, and wavefunctions, $\Psi_{n}(r)$, of the quantum-confined levels for a spherically symmetric lead chalcogenide SNs (e.g., quantum dots) can be found by applying boundary conditions $\left.\Psi(r)\right|_{|r|=a}=0$, where $a$ is the quantum dot radius. The entire procedure of finding such quantum-confined energies and wavefunctions is described in great detail in the seminal paper by Kang and Wise [22], and briefly outlined in Appendix B, putting particular emphasis on the symmetry considerations.

Once the wavefunctions (four-spinors) and the corresponding energies are obtained for the quantumconfined levels within the conduction $(E>0)$ and the valence $(E<0)$ bands, the electron-EMF coupling can be calculated. Operator for the lowest-order electron-EMF interaction, Eq. (13), becomes

$$
F(\mathbf{r}, \mathbf{p}, t)=\frac{e}{c}\left[\begin{array}{cc}
\frac{\mathbf{p} \cdot \mathbf{A}(\mathbf{r}, t)+\mathbf{A}(\mathbf{r}, t) \cdot \mathbf{p}}{2 m_{c}} I_{2} & \frac{P}{m}(\boldsymbol{\sigma} \cdot \mathbf{A}(\mathbf{r}, t)) \\
\frac{P}{m}(\boldsymbol{\sigma} \cdot \mathbf{A}(\mathbf{r}, t)) & -\frac{\mathbf{p} \cdot \mathbf{A}(\mathbf{r}, t)+\mathbf{A}(\mathbf{r}, t) \cdot \mathbf{p}}{2 m_{v}}
\end{array}\right]
$$

where $I_{4}$ is the $4 \times 4$ unit matrix. Vector and scalar potentials in velocity and length gauges are given by Eqs. (6) and (7), respectively. For definiteness we assume $\mathbf{A}_{0}=A_{0} \hat{\mathbf{z}}$, where $\hat{\mathbf{z}}$ is the unit vector along z-axis. Then, amplitudes of photoinduced transitions between the eigenstates of Hamiltonian (25) are then encoded by operators

$$
f_{1}(\mathbf{r}, \mathbf{p})=\frac{e}{c}\left[\begin{array}{cc}
\frac{p_{z}}{m_{c}} I_{2} & \frac{P}{m} \sigma_{z} \\
\frac{P}{m} \sigma_{z} & -\frac{p_{z}}{m_{v}} I_{2}
\end{array}\right],
$$

and

$$
f_{2}(\mathbf{r}, \mathbf{p})=e z \frac{\omega_{0}}{c} I_{4}
$$

so that $F=A_{0} f_{1} \sin \left(\omega_{0} t\right)$ and $F=A_{0} f_{2} \cos \left(\omega_{0} t\right)$ for the velocity and length gauge, respectively.

In what follows we will focus on the lowest-energy photoinduced transition, which is most relevant for photoluminescence. This is the transition between the highest energy quantum-confined electronic state in 
the valence band and the lowest energy quantum-confined state in the conduction band. These states are designated as $\left(n, J, J_{z}, \pi\right)_{v}=(1,1 / 2, \pm 1 / 2,1)_{v}$ and $\left(n, J, J_{z}, \pi\right)_{c}=(1,1 / 2, \pm 1 / 2,-1)_{c}$, respectively, where $n$ is the radial quantum number, $J$ and $J_{z}$ are the total angular momentum and its projection onto z-axis, respectively, and $\pi$ is parity [22]. The respective wavefunctions are $\Psi_{-1}$ and $\Psi_{1}$. Each of these states is doubly degenerate with respect to the projection of the total angular momentum. However, this projection is conserved during the transition since we chose $\mathbf{A}_{0}=A_{0} \hat{\mathbf{z}}$. Henceforth, we consider the photoinduced transition between states with $J_{z}=+1 / 2$ for definiteness. As previously, we can define an effective energydependent Kane momentum as (substituting $\hbar \omega_{0}$ with transition energy $E$ )

$$
\tilde{P}_{i}(E)=\frac{c m}{e}\left\langle\Psi_{1}\left|f_{i}\right| \Psi_{-1}\right\rangle, i=1,2 .
$$

As discussed in Sec. 3, the choice of gauge does not affect the transition intensities. We tested numerically that this is indeed the case for the two gauge choices, Eqs. (27) and (28), up to numerical round-off errors, so that

$$
\tilde{P}^{2}(E)=\tilde{P}_{1}^{2}(E)=\tilde{P}_{2}^{2}(E) .
$$

The normalized transition intensity, $\tilde{P}^{2}(E) / P^{2}$, is plotted in Fig. 1 for PbSe and PbS with material parameters taken from Ref. [22]. The observed behavior - transition intensity decreases with increasing degree of quantum confinement - is qualitatively similar to that observed for the two-band Kane model in Sec. 4.2. In particular, making Hamiltonian (25) dimensionless similarly to how it was done in the beginning of Sec. 4, one obtains the dimensionless Kane momentum matrix element as $\alpha=2 P \sqrt{\frac{4 m_{*}}{m^{2} E_{g}}}$, where $m_{*}$ is the characteristic far-band contribution to carrier effective masses. Substituting numerical values for PbSe and $\mathrm{PbS}$ parameters to this expression one obtains $\alpha \approx 1-2$. The intermediate regime of Kane momentum renormalization does not appear for such small values of $\alpha$ in the spatially confined Kane model, see red and black curves in Fig. 4(a). Based on this, one would expect a simple monotonic featureless decrease of $\tilde{P}^{2}(E) / P^{2}$ as a function of $E$ for lead chalcogenide QDs, starting from $\tilde{P}^{2}(E)=P^{2}$ at low energies and converging to some finite value $0<\tilde{P}^{2}(E) / P^{2}<1$ at $E \rightarrow \infty$. This is exactly what is observed in Fig. 1 .

Finally, we would like to discuss the dependence of the renormalized Kane momentum and the renormalized transition dipole moment on energy. Combining Eqs. (28) and (29) with the direct consequence of gauge invariance, Eq. (30), one obtains

$$
\tilde{P}^{2}(E) \propto E^{2} \tilde{d}^{2}(E)
$$

where $\tilde{d}(E)=\left|\left\langle\Psi_{1}|e z| \Psi_{-1}\right\rangle\right|$ is the dipole moment corresponding to the lowest-energy transition. Eq. (31) is exactly the relation between the effective transition dipole moment and the effective Kane momentum we guessed in Introduction. In particular, this relation guarantees that Eqs. (1) and (2) produce exactly the same spontaneous emission rates. On the other hand, since $\tilde{P}(E)$ already decays with $E$, as is seen in Fig. 1, Eq. (31) implies that $\tilde{d}(E)$ decays rapidly with $E$. This suggests that the renormalized dipole moment is not especially convenient to use when describing the strength of electron-EMF interaction in narrow-gap SNs. Effective Kane momentum $\tilde{P}(E)$ depends on $E$ much less strongly and, perhaps, could even be approximated by a constant within an experimentally relevant range of energies.

\section{Discussion}

In this section we will discuss two example approaches of calculation of the electron-EMF coupling strength, which ignore the effect of band mixing, and clarify exactly why they produce inaccurate results.

\subsection{Velocity gauge}

The first approach is based on the velocity gauge, Eq. (6), where the scalar potential vanishes exactly. The typical calculation of coupling strength in this gauge, which neglects band mixing, proceeds as follows. Kane momentum matrix element in Hamiltonian (25) is set to zero, thus neglecting band mixing. At these 


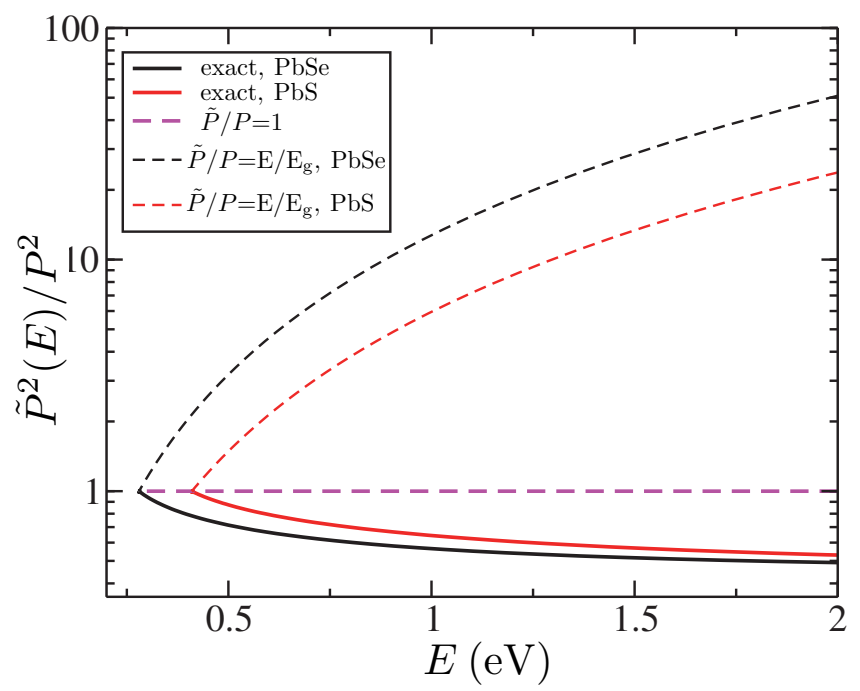

Figure 5: Energy-dependent normalized transition intensity for the lowest optical transition in PbSe and PbS QDs. Exact numerical results are given by solid lines. Dashed magenta line corresponds to neglecting band mixing in the velocity gauge. Dashed black and red lines correspond to neglecting band mixing in the length gauge.

conditions, finding size-quantized levels becomes very simple since the entire problem reduces to a singleband effective mass approximation. Specifically, the wavefunctions corresponding to the lowest conduction band and the highest valence band states with total angular momentum projection of $J_{z}=+1 / 2$ are

$$
\begin{gathered}
\Psi_{1} \propto\left[j_{0}(\kappa r), 0,0,0\right]^{T}, \\
\Psi_{-1} \propto\left[0,0, j_{0}(\kappa r), 0\right]^{T},
\end{gathered}
$$

respectively, where $\kappa=\pi / a$ and $j_{0}(x)=\sin x / x$ is the zeroth-order spherical Bessel function of the first kind. These wavefunctions can now be substituted into Eq. (29) with $f_{i}=f_{1}$ to evaluate the coupling between them due to interaction with EMF. The result, $\tilde{P}(E) \equiv P$, is shown as a horizontal dashed magenta line in Fig. 5. Neglecting the band mixing in the velocity gauge does thus result in the absence of the Kane momentum renormalization. Exact results, obtained in the previous section for $\mathrm{PbSe}$ and $\mathrm{PbS}$ are shown by solid black and solid red lines, respectively. Comparing these approximate and exact results one can conclude that the effect of band mixing on the strength of the electron-EMF coupling can not in general be disregarded except for very large QDs where the energy of the lowest optical transition approaches the bulk bandgap energy $E_{g}$. However, since the renormalized $\tilde{P}(E)$ does not deviate from $P$ by more than a factor of $\sim 2$ for $\mathrm{PbSe}$ and $\mathrm{PbS}$, one might be satisfied with assuming $\tilde{P}(E) \approx P$ depending on the required accuracy.

However, what is not immediately clear at the first glance is that the considered approach is also not gauge-invariant. As discussed in Sec. 3, the gauge invariant formulation could be obtained from Hamiltonian (25) via substitution $\mathbf{p} \rightarrow \mathbf{p}+\frac{e}{c} \mathbf{A}(\mathbf{r}, t)$ and adding the scalar potential. The resulting Hamiltonian is the polynomial of the second order with respect to the vector potential. To arrive at the approximate approach just described above, one needs to set $P$ to zero, but only in the polynomial terms that are of zeroth-order with respect to $\mathbf{A}(\mathbf{r}, t)$, which represent the material part of the Hamiltonian. Obviously this breaks the gauge invariance since the resulting approximate Hamiltonian cannot be reduced to one where the vector potential only enters via $\mathbf{p}+\frac{e}{c} \mathbf{A}(\mathbf{r}, t)$. To illustrate the danger of breaking the gauge invariance by an example, we start from the velocity gauge, Eq. (6), and perform the gauge transformation, Eq. (5), with $f(\mathbf{r}, t)=A_{1} z \sin \left(\omega_{0} t\right)$ where $A_{1}$ is an arbitrary value. The resulting vector and scalar potentials are $\mathbf{A}(\mathbf{r}, t)=$ $\left(A_{0}+A_{1}\right) \hat{\mathbf{z}} \sin \left(\omega_{0} t\right)$ and $\varphi(\mathbf{r}, t)=-\frac{\omega_{0}}{c} z \cos \left(\omega_{0} t\right)$. The scalar potential does not couple wavefunctions $\Psi_{1}$ and $\Psi_{-1}$ given by Eq. (32) since it always enters the Hamiltonian matrix strictly diagonally, see Eq. (12). The 
amplitude of the vector potential upon the gauge transformation, $A_{0}+A_{1}$, is arbitrary and therefore the transition intensity could be made as large as desired. Therefore, that the velocity gauge-based approach described in this subsection does yield results that are different from the exact ones by no more than a factor of $\sim 2$ is solely due to the fortunate choice of the gauge. On the other hand, the inclusion of the band mixing would allow the scalar potential to couple $\Psi_{1}$ and $\Psi_{-1}$, resulting in a matrix element opposite in sign to that produced by the vector potential, thus compensating the arbitrary increase in the amplitude of the vector potential.

\subsection{Length gauge}

Another approach to evaluate the strength of the electron-EMF coupling is based on the length gauge, Eq. (7). Calculation of the coupling strength in this gauge could proceed as follows. The full microscopic wavefunctions are written as $\Psi_{1}(\mathbf{r}) \propto j_{0}(\kappa r) \chi_{c k}(\mathbf{r})$ and $\Psi_{-1}(\mathbf{r}) \propto j_{0}(\kappa r) \chi_{v k}(\mathbf{r})$, i.e., combining Eqs. (32) and (9). Coupling between these two wavefunctions is then given by

$$
\left\langle\Psi_{1}\left|f_{1}\right| \Psi_{-1}\right\rangle=\frac{e \omega_{0}}{c} \int d \mathbf{r} \Psi_{1}^{*}(\mathbf{r}) z \Psi_{-1}(\mathbf{r}) .
$$

This integral can be approximately evaluated assuming that the envelope functions (i.e., spherical Bessel functions) change much more slowly with $\mathbf{r}$ than the Bloch functions $\chi_{c(v) k}(\mathbf{r})$. At these conditions, Eq. (29) becomes

$$
\tilde{P}(E)=\frac{m E}{e \hbar} d
$$

where $E$ is the transition energy and

$$
d=e\left|\int_{u c} d \mathbf{r} \chi_{c k}^{*}(\mathbf{r}) z \chi_{v k}(\mathbf{r})\right|
$$

is the transition dipole moment of a single unit cell, corresponding to the transition between the valence and the conduction bands. Taking into account the relation $d=\frac{e \hbar P}{m E_{g}}[16,15]$, one obtains

$$
\tilde{P}^{2}(E) / P^{2}=\left(E / E_{g}\right)^{2}
$$

that is the effective Kane momentum grows rapidly with energy, as is shown by dashed black and red lines for $\mathrm{PbSe}$ and $\mathrm{PbS}$, respectively, in Fig. 5. The obtained results are generally very different from the exact ones except when $E \approx E_{g}$. The stark disagreement with the exact results can be understood from the following considerations. As it was discussed in Sec. 3, the band mixing is present in Hamiltonian (25) not only explicitly via the off-diagonal terms proportional to $P$, but also implicitly via $m_{c}$ and $m_{v}$, which come about from mixing with far-bands. Since this mixing is present, it is not correct anymore to assume that an envelope function could be converted to its microscopic representation using Eq. (9), as discussed in Sec. 3. To clarify this, we consider an example where the contribution of far bands can be neglected whatsoever. Then, one has to have $m_{c}=m$ and $m_{v}=-m$ in Eq. (25). In the absence of band mixing, the quantum confinement would just shift all the levels by the same amount of energy, so $E \equiv E_{g}$. At these conditions, $\tilde{P}(E) \equiv P$, i.e., identical to the result obtained for the velocity gauge.

To conclude this section, we would like to reiterate that there is a single gauge-invariant approach to account for the electron-EMF interaction within the envelope function approximation. This approach consists of transforming Hamiltonian (10) into Hamiltonian (12) via the minimal-coupling procedure. Other approaches, if not reducible to Eq. (12), break the gauge invariance and, therefore, produce unphysically gauge-dependent experimentally measurable quantities such as photoinduced transition rates. This section gives two most typical examples of such approaches where gauge invariance is broken. Other examples could be found in literature. For example, a mixture of the two provided examples was used in Ref. [22]. Specifically, the full microscopic wavefunction was "restored" by disregarding the mixing with far-bands, which broke the gauge invariance. The transition intensity was then evaluated within the velocity gauge, producing Eq. (30) in Ref. [22]. That the obtained expression is incorrect can already be recognized by 
observing that it does not include far-band contributions to effective masses as parameters. The correct coupling operator, Eq. (27), was obtained from the gauge-invariant Eq. (13), and is seen to contain those far-band contributions to effective masses.

\section{Conclusion}

In this work, we have investigated the effect of band mixing within the envelope function approximation on the strength of the electron-EMF interaction in SNs. Often, the strength of this interaction is considered to be given by the Kane momentum matrix element, which is the property of the bulk semiconductor and, therefore, is independent of size-quantization effects in e.g., semiconductor quantum dots. In this work we demonstrate that even though such an approximation could be relatively accurate in wide-bandgap semiconductors (e.g., CdSe, CdS), it breaks down in narrow-gap semiconductors (e.g., PbSe, PbS). In particular, we observe that neglecting the band mixing effects can lead to overestimation of photoinduced transition or spontaneous emission rates by a factor of $\sim 2$ for PbSe and $\mathrm{PbS}$ quantum dots.

To obtain insight into how exactly band mixing affects the strength of electron-EMF coupling, we first analyzed a simple possible model with band mixing - two-band 1D Kane model. The effect could be seen most transparently in the continuous Kane model, i.e., where no size quantization was present. On a very qualitative approximate level, one can think that both (i) band mixing terms of the envelope function Hamiltonian and (ii) the external EMF do perform the same function - they couple electronic states from different bands (e.g., valence and conduction). Then, when band mixing terms are weak, e.g., when energies are very close to band edges, EMF mixes bands most efficiently resulting in the strong electron-EMF coupling. On the other hand, when the effect of mode mixing terms is significant (i.e., higher energies), the conduction and valence states become strongly mixed within the envelope function formalism. Under these conditions, the external EMF cannot mix such states any further since they are already strongly mixed into symmetric and antisymmetric linear combinations of conduction and valence band states. This results in a diminished strength of the electron-EMF coupling. The picture becomes much more involved once the spatial quantization is present, since a single state from a band can now couple to multiple states within the other band. Nevertheless, the introduced qualitative picture holds if we substitute energies of the continuous electronic states with discrete quantum confinement energies in SNs. Specifically, the electron-EMF coupling is strongest in large SNs, which corresponds to small confinement energies, and becomes weaker in smaller SNs where confinement energies become larger.

It turns out that even though the phenomenon of renormalization of electron-EMF interaction by band mixing could be rather involved physically, it can nevertheless be quantified by a single energy-dependent function - an effective energy-dependent Kane momentum, $\tilde{P}(E)$. Such a function in general depends on the material parameters (e.g., bulk band gap, effective masses) and on the SN shape. Fig. 1 shows our numerical results for the effective energy-dependent Kane momenta for PbSe and $\mathrm{PbS}$ spherically symmetric SNs (i.e., quantum dots). This renormalized Kane momenta can now be used in all the formulae previously developed to treat the electron-EMF interaction in semiconductor quantum dots in the absence of band mixing. For instance, the familiar expression for rate of spontaneous emission, Eq. (1), can be modified to properly account for the band mixing by a simple substitution $P \rightarrow \tilde{P}(E)$, where $\tilde{P}(E)$ for $\mathrm{PbSe} / \mathrm{PbSe} \mathrm{QDs}$ can be taken from Fig. 1. Other quantities related to the electron-EMF coupling strength, e.g., cross sections of absorption and stimulated emission, are linearly related to the rate of spontaneous emission by virtue of the Einstein coefficients. Therefore, the conventional bulk-like expressions for such quantities could also be modified to account for band mixing via the same substitution.

Finally, we would like to emphasize that a consistent treatment of the electron-EMF coupling must necessarily preserve the gauge invariance. For example, the apparent contradiction between Eqs. (1) and (2) originates from approximations that break the gauge invariance of the $k \cdot p$ model. Two typical examples of calculations of the electron-EMF coupling where the gauge invariance is broken are presented and discussed in Sec. 6 . The requirement of the gauge invariance is not unique to the $k \cdot p$ model, as it is also very important in tight-binding [34, 35] and pseudopotential-based calculations [36, 37]. 


\section{Conflict of interest}

There is no conflict of interest.

\section{Acknowledgements}

We are grateful to Nikolay Makarov and Oleksandr Isaienko for useful discussion. K.A.V. was supported by the Center for Advanced Solar Photophysics (CASP), an Energy Frontier Research Center funded by the Office of Basic Energy Sciences, Office of Science, US Department of Energy (DOE).

\section{Appendix A. Spatially confined 1D Kane model}

The Hamiltonian of the 1D Kane model in the absence of the EM fields is given by Eq. (18). The spatial confinement is introduced by requiring the wavefunction to vanish at the "surface", i.e., $\Psi(x= \pm L / 2)=0$, where $L$ is the size of the 1D "quantum dot". The general approach to finding the eigenstates of such a problem is as follows. First, bulk solutions (i.e., without boundary conditions) are found for a given energy $E$. Since the Hamiltonian is a $2 \times 2$ matrix quadratic with respect to momentum $p$, there is four bulk solutions, each represented by a two-component spinor. Specifically, there are two propagating and two evanescent plane waves at $|E|>1$, whereas all the waves are evanescent at $|E|<1$. In what follows, we will only consider the former case. Characteristic equation for Hamiltonian (18) is

$$
E^{2}=\left(1+p^{2}\right)^{2}+\alpha^{2} p^{2}
$$

Solving this equation with respect to momentum produces

$$
p^{2}=-\left(1+\alpha^{2} / 2\right) \pm \sqrt{E^{2}+\left(1+\alpha^{2} / 2\right)^{2}-1} .
$$

This yields two propagating solutions (i.e., real momenta)

$$
p_{1,2}= \pm k= \pm\left(-\left(1+\alpha^{2} / 2\right)+\sqrt{E^{2}+\left(1+\alpha^{2} / 2\right)^{2}-1}\right)^{1 / 2}
$$

and two evanescent ones (imaginary momenta)

$$
p_{3,4}= \pm i \kappa= \pm i\left(\left(1+\alpha^{2} / 2\right)+\sqrt{E^{2}+\left(1+\alpha^{2} / 2\right)^{2}-1}\right)^{1 / 2} .
$$

The corresponding spinors are (non-normalized)

$$
\Psi_{i}=\left[\begin{array}{c}
\alpha p_{i} \\
E-1-p_{i}^{2}
\end{array}\right] e^{i p_{i} x}, i=1, \ldots, 4 .
$$

To find a spatially confined solution, the boundary conditions have to be satisfied by a linear combination of these four spinors. The problem can be simplified if one explicitly accounts for the parity symmetry. Specifically, both Hamiltonian (18) and the boundary conditions are symmetric with respect to the parity transformation, $\hat{\pi}=-\sigma_{z} \hat{P}$, where $\hat{P} \Psi(x)=\Psi(-x)$ is the conventional spatial inversion. Under this condition, it is convenient to first construct specific-parity linear combinations of spinors (A.5). Then, matching boundary conditions for such symmetrized spinors involves linear combinations of only two spinors for each parity. The odd-parity $(\hat{\pi} \Psi=-\Psi)$ spinor can be written as

$$
\Psi(x)=A\left[\begin{array}{c}
\alpha k \cos (k x) \\
i\left(E-1-k^{2}\right) \sin (k x)
\end{array}\right]+B\left[\begin{array}{c}
\alpha \kappa \cosh (\kappa x) \\
i\left(E-1+\kappa^{2}\right) \sinh (\kappa x)
\end{array}\right],
$$

Coefficients $A$ and $B$ has to found so that $\Psi(+L / 2)=0$, and then $\Psi(-L / 2)=0$ is satisfied automatically. Matching this boundary condition results in the following characteristic equation

$$
k\left(E-1+\kappa^{2}\right) \tanh (\kappa L / 2)=\kappa\left(E-1-k^{2}\right) \tan (k L / 2) .
$$


The even-parity $(\hat{\pi} \Psi=\Psi)$ spinor reads as

$$
\Psi(x)=A\left[\begin{array}{c}
\alpha k \sin (k x) \\
-i\left(E-1-k^{2}\right) \cos (k x)
\end{array}\right]+B\left[\begin{array}{c}
\alpha \kappa \sinh (\kappa x) \\
i\left(E-1+\kappa^{2}\right) \cosh (\kappa x)
\end{array}\right] .
$$

The characteristic equation, resulting from the boundary conditions, is

$$
\kappa\left(E-1-k^{2}\right) \tanh (\kappa L / 2)=-k\left(E-1+\kappa^{2}\right) \tan (k L / 2) .
$$

In general, such transcendental characteristic equations have to be solved numerically for eigenenergies $E$. However, some general properties of such solutions can be established right away. First, at $E>1$ both $\left(E-1-k^{2}\right)$ and $\left(E-1+\kappa^{2}\right)$ are always positive. This means that $\tan (k L / 2)$ has to be positive in Eq. (A.7) and negative in Eq. (A.9). Second, $\tan (k L / 2)$ does always vanish at $k L / 2=\pi n, n=0,1,2, \ldots$ and always diverges to positive or negative infinity when $k L / 2$ approaches $\pi(n+1 / 2)$ from the left or right, respectively. These two observations imply that Eq. (A.7) does always have one and only one solution within each interval $k L / 2 \in \pi(n, n+1 / 2), n=0,1,2, \ldots$. Similarly, Eq. (A.7) does always have one and only one solution within each interval $k L / 2 \in \pi(n+1 / 2,2 n), n=0,1,2, \ldots$. Therefore, positive-energy states of the spatially confined 1D Kane model have alternating parities when sorted with respect to their energies, with the lowest-energy state being the odd one.

Negative-energy solutions $(E<-1)$ can be found similarly. However, It is more convenient to exploit the charge conjugation symmetry of Hamiltonian (18). Specifically, it can be straightforwardly demonstrated that $\mathrm{CH}_{0} \mathrm{C}^{\dagger}=-H_{0}$ and $C \hat{\pi} C^{\dagger}=-\hat{\pi}$, where $C=-i \sigma_{y}$ is the charge conjugation operator. These identities imply that this transformation flips the parity and the sign of the energy. More specifically, we can enumerate all the solutions of the spatially confined 1D Kane problem according to their energies: ... $<E_{-2}<E_{-1}<$ $0<E_{1}<E_{2}<\ldots$. Then, the charge conjugation symmetry yields: (i) $E_{-n}=-E_{n}$, (ii) $\Psi_{-n}(x)=C \Psi_{n}(x)$, and (iii) the parity is $\pi_{n}=(-1)^{|n+1 / 2|-1 / 2}$. In particular, the energies of the two band edge states are related as $E_{-1}=-E_{1}$ with the negative- (positive) energy state being of the even (odd) parity. In what follows, we consider three limiting cases, where analytical expressions for energies and wavefunctions of these band edge states can be obtained explicitly.

\section{Appendix A.1. Weak confinement}

The weak confinement is realized at $L \gg 1, \alpha$. At these conditions, the energy of the positive-energy band edge state is $0<E_{1}-1 \ll 1$ and Eqs. (A.3) and (A.4) reduce to $k=\sqrt{\frac{E_{1}-1}{1+\alpha^{2} / 2}}$ and $\kappa=\sqrt{2\left(1+\alpha^{2} / 2\right)}$. Then, the solution of Eq. (A.7) is $E_{1}=1+\left(1+\frac{\alpha^{2}}{2}\right) \tilde{p}_{1}^{2}$, where the effective momentum corresponding to the band edge states is denoted by $\tilde{p}_{1}=\pi / L$. The corresponding wavefunction is

$$
\Psi_{1}(x)=\sqrt{\frac{2}{L}}\left[\begin{array}{c}
\cos \left(\tilde{p}_{1} x\right) \\
0
\end{array}\right] .
$$

The energy and the wavefunction of the negative-energy band edge state is obtained using the charge conjugation symmetry as $E_{-1}=-E_{1}$ and

$$
\Psi_{-1}(x)=C \Psi_{1}(x)=\sqrt{\frac{2}{L}}\left[\begin{array}{c}
0 \\
\cos \left(\tilde{p}_{1} x\right)
\end{array}\right] .
$$

The normalized transition intensity (24) can then be evaluated as

$$
\left|\left\langle\Psi_{1}\left|f_{1}\right| \Psi_{-1}\right\rangle^{2}\right| / \alpha^{2}=1
$$

\section{Appendix A.2. Intermediate confinement}

In the intermediate regime, $\alpha^{-1} \ll L \ll \alpha$, the energy of the lowest positive-energy state is seen in Fig. 3 to be $E_{1} \sim \alpha / L$, so that $1 \ll E_{1} \ll \alpha^{2}$. Under these conditions, Eqs. (A.3) and (A.4) reduce to $k_{1}=E_{1} / \alpha$ 
and $\kappa_{1}=\alpha$, respectively. Substituting these expressions into Eq. (A.7) one obtains $\tan \left(E_{1} L / 2 \alpha\right)=1$, resulting in

$$
E_{1}=\alpha \tilde{p}_{1} / 2
$$

where, again, $\tilde{p}_{1}=\pi / L$. The corresponding wavefunction is

$$
\Psi_{1}(x)=\frac{1}{\sqrt{L}}\left[\begin{array}{c}
\cos \left(\tilde{p}_{1} x / 2\right)-\sqrt{2} e^{-\alpha L / 2} \cosh (\alpha x) \\
i \sin \left(\tilde{p}_{1} x / 2\right)-i \sqrt{2} e^{-\alpha L / 2} \sinh (\alpha x)
\end{array}\right] .
$$

We wish to note here that the naive recipe for performing the 1D spatial quantization (e.g., how it is done within the parabolic effective mass approximation) is to evaluate the energy of the spatially confined state by substituting the effective momentum $\tilde{p}_{1}$ into the bulk dispersion relation, Eq. (A.1). Within the considered intermediate regime, such a procedure would yield $E_{1}=\alpha \tilde{p}_{1}$, which differs from the correct expression, Eq. (A.13), by a numerical factor. The inapplicability of the naive quantization recipe is further emphasized by an observation that even though the diagonal part of Hamiltonian (18) could be neglected in the continuous case when $\alpha^{-1} \ll p_{1} \ll \alpha$, such an approximation would be too crude in the spatially quantized case. Indeed, completely discarding the diagonal part yields a Hamiltonian matrix which is linear with respect to the momentum operator. For such a Hamiltonian, there is only a single linearly independent bulk solution per specific parity, which is not enough to satisfy the boundary conditions. In Eq. (A.14), the boundary conditions are satisfied due to the presence of the evanescent tails of the wavefunction, which are only significant near the edges $(x \approx \pm L / 2)$. These tails can only be obtained if Hamiltonian terms proportional to $p^{2}$ are taken into account. That the presence of these evanescent tails is essential invalidates the naive 1D quantization recipe and results in the spatial confinement energy, Eq. (A.13), that is a factor of two lower than the naive result, $E_{1}=\alpha \tilde{p}_{1}$.

The wavefunction for the negative-energy band edge state is obtained via the charge conjugation transformation

$$
\Psi_{-1}(x)=C \Psi_{1}(x)=\frac{1}{L}\left[\begin{array}{c}
-i \sin \left(\tilde{p}_{1} x / 2\right)+i \sqrt{2} e^{-\alpha L / 2} \sinh (\alpha x) \\
\cos \left(\tilde{p}_{1} x / 2\right)-\sqrt{2} e^{-\alpha L / 2} \cosh (\alpha x)
\end{array}\right] .
$$

The normalized transition intensity can then be evaluated analytically to yield

$$
\left|\left\langle\Psi_{1}\left|f_{1}\right| \Psi_{-1}\right\rangle\right|^{2} / \alpha^{2}=\frac{4}{\pi^{2}} \approx 0.4053
$$

\section{Appendix A.3. Strong confinement}

In the limit of the very strong confinement, $L \ll 1, \alpha^{-1}$, one obtains $k^{2}=\kappa^{2}=E_{1} \gg 1, \alpha$. The prefactor in the l.h.s of Eq. (A.7) is then much larger than the prefactor in the r.h.s., and, therefore, the tangent function has to be very large to compensate for this mismatch. This results $E_{1}=\tilde{p}_{1}^{2}$ for the lowest positive-energy state. The corresponding wavefunction is

$$
\Psi_{1}(x)=A\left[\begin{array}{l}
\alpha \tilde{p}_{1} \cos \left(\tilde{p}_{1} x\right) \\
i \frac{\alpha^{2}}{2} \sin \left(\tilde{p}_{1} x\right)
\end{array}\right]+B\left[\begin{array}{l}
\alpha \tilde{p}_{1} \cosh \left(\tilde{p}_{1} x\right) \\
2 i \tilde{p}_{1} \sinh \left(\tilde{p}_{1} x\right)
\end{array}\right],
$$

where coefficients $A$ and $B$ are found as follows. The requirement of vanishing lower component of the spinor at $x=L / 2$ yields $B / A=-\alpha\left[4 \tilde{p}_{1}^{2} \sinh (\pi / 2)\right]^{-1}$. For this ratio of coefficients, $A \cos \left(\tilde{p}_{1} x\right)$ is always much larger in magnitude than $B \cosh \left(\tilde{p}_{1} x\right)$ within the upper spinor component, so that the latter term can be safely discarded. The resulting normalized wavefunction reads as

$$
\Psi_{1}(x)=\sqrt{\frac{2}{L}}\left[\begin{array}{c}
\cos \left(\tilde{p}_{1} x\right) \\
\frac{i \alpha}{2 \tilde{p}_{1}}\left[\sin \left(\tilde{p}_{1} x\right)-\frac{\sinh \left(\tilde{p}_{1} x\right)}{\sinh (\pi / 2)}\right]
\end{array}\right] .
$$

The wavefunction for the negative-energy band edge state is obtained using the charge conjugation transformation

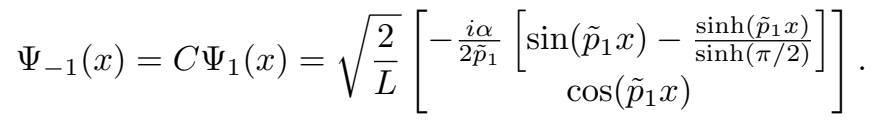


The transition matrix element can then be evaluate analytically to yield

$$
\left|\left\langle\Psi_{1}\left|f_{1}\right| \Psi_{-1}\right\rangle\right|^{2} / \alpha^{2}=\frac{[4 \operatorname{coth}(\pi / 2)-\pi]^{2}}{\pi^{2}} \approx 0.1507 .
$$

\section{Appendix B. Kang-Wise problem}

Spherically symmetric Dimmock Hamiltonian is given by Eq. (25). Electron and hole size-quantized levels in SN are found as a solution of a corresponding stationary Schrödinger equation subject to boundary conditions. Specifically, size-quantized levels in a quantum dot (QD) are found by requiring the wavefunction to be non-singular inside the QD and to vanish at the QD surface, $\left.\Psi(r)\right|_{|r|=a}=0$, where $a$ is the QD radius. The standard procedure of finding size-quantized levels via matching the boundary conditions is as follows [38]. First, all the linearly independent eigenfunctions of Hamiltonian (25) of specific energy and symmetry are found. Second, a linear combination of these solutions with unknown coefficients is required to vanish at the QD surface - this condition yields a secular equation. Solving this secular equation results in the energies of the size-quantized states. Below we demonstrate how to employ symmetry considerations to construct the exact ansatz for a wavefunction and thus simplify the solution of the problem.

The symmetries of the Hamiltonian (25) and of the boundary conditions are given by the following operators:

1. Parity

$$
\Pi_{4}=\left[\begin{array}{cc}
-\pi I_{2} & 0 \\
0 & \pi I_{2}
\end{array}\right]
$$

where $\pi$ is a coordinate inversion operator $(\mathbf{r} \rightarrow-\mathbf{r})$. The global sign of the operator is chosen to represent the parity of the total microscopic wavefunction, since the conduction band Bloch function is odd, and the valence band Bloch function is even [22]. It is clear, however, that as long as $k \cdot p$ Hamiltonian is specified, parity is its internal property and the original parity of the Bloch functions are irrelevant. For example, even if a certain material does not have any inversion symmetry, but a corresponding effective Hamiltonian could be written as Eq. (25), then the parity operator (B.1) could be associated with it.

2. Total angular momentum and its projection

$$
J_{4, z}=\left[\begin{array}{cc}
J_{z} & 0 \\
0 & J_{z}
\end{array}\right], J_{4}^{2}=\left[\begin{array}{cc}
J^{2} & 0 \\
0 & J^{2}
\end{array}\right]
$$

Here, the operator of the total angular momentum is given by $\mathbf{J}=\mathbf{L} I_{2}+\boldsymbol{\sigma} / 2$, where $\mathbf{L}$ is the operator of the orbital momentum.

3. Square of the momentum

$$
p_{4}^{2}=p^{2} I_{4}
$$

where $I_{4}$ is the $4 \times 4$ unit matrix.

All these operators commute with each other each other and the Hamiltonian, so it is possible to find wavefunctions of Hamiltonian (25), which would satisfy all the symmetries above. Square of the momentum is not a conventional symmetry operator, but it can be straightforwardly shown to commute with the Hamiltonian and will be of use in what follows. Importantly, all the symmetry operators are block-diagonal, i.e., all these symmetries are applicable not only to the entire four-spinors, but also to the upper or lower bi-spinor separately. Specifically, the fact that $J_{z}, J^{2}$ and $\pi I_{2}$ have to be conserved for an upper and lower bi-spinor independently, results in that the angular part of a bi-spinor of a specific parity can be represented as a spin spherical harmonic [39]

$$
\chi_{j l m}(\Omega)=\left[\begin{array}{c}
C(l, m-1 / 2 ; 1 / 2,1 / 2 ; j, m) Y_{l, m-1 / 2}(\Omega) \\
C(l, m+1 / 2 ; 1 / 2,-1 / 2 ; j, m) Y_{l, m+1 / 2}(\Omega)
\end{array}\right],
$$


where $j, l$ and $m$ are the total angular momentum, orbital momentum of the coordinate part of the wavefunction, and the projection of the total angular momentum, respectively. Clebsh-Gordan coefficients are denoted by $C\left(j_{1}, m_{1} ; j_{2}, m_{2} ; j, m\right)$. The parity of this bi-spinor with respect to $\mathbf{r} \rightarrow-\mathbf{r}$ is given by $(-1)^{l}$. For a given $j$ and $m$, there are only two possible bi-spinors with orbital momenta $l=j \pm 1 / 2$.

To obtain a full bi-spinor, one has to multiply its angular part by a radial part, $f_{l}(r)$. Since bi-spinors have to be eigenfunctions of operator $p^{2} I_{2}$, we have

$$
p^{2} \chi_{j l m}(\Omega) f_{l}(r)=-\chi_{j l m}(\Omega)\left[\frac{\partial^{2}}{\partial r^{2}}+\frac{2}{r} \frac{\partial}{\partial r}-\frac{l(l+1)}{r^{2}}\right] f_{l}(r)=\kappa^{2} \chi_{j l m}(\Omega) f_{l}(r),
$$

where $\kappa^{2}$ denotes the eigenvalue corresponding to operator $p^{2}$. The solution of this equation is the spherical Bessel function of the first kind, $f_{l}(r)=j_{l}(\kappa r)$ [40]. Spherical Bessel function of the second kind is also the solution, but it is not suitable for the expansion of the final wavefunction since it is singular at $r=0$.

Operator $\boldsymbol{\sigma} \cdot \mathbf{p}$ flips the parity of the bi-spinor, does not change $j$ or $m$, and commutes with $p^{2}$. Therefore, operator $\boldsymbol{\sigma} \cdot \mathbf{p}$ can only transform a bi-spinor with quantum numbers $(j, l, m)$ to the one with with quantum numbers $\left(j, l^{\prime}, m\right)$, where $l^{\prime}=2 j-l$. It is thus expected that the radial part of operator $\boldsymbol{\sigma} \cdot \mathbf{p}$ has to be a raising or lowering operator for the spherical Bessel functions. Indeed, if one combines $j$ and $l$ into a single quantum number $k=\mp(j+1 / 2)$ for $j=l \pm 1 / 2$, then spherical spinors can be written as $\chi_{k m}(\Omega)$ and one has $[41]$

$$
\boldsymbol{\sigma} \cdot \mathbf{p} \chi_{k m}(\Omega)=i\left(\frac{\partial}{\partial r}+\frac{k+1}{r}\right) \chi_{-k m}(\Omega) .
$$

The radial operator in parentheses becomes $A_{l}^{-}$for positive $k(k=l)$ and $-A_{l}^{+}$for negative $k(k=-l-1)$, where the raising and lowering operators for the spherical Bessel functions are defined as

$$
\begin{aligned}
& A_{l}^{-} j_{l}(r)=\left(\frac{\partial}{\partial r}+\frac{l+1}{r}\right) j_{l}(r)=j_{l-1}(r), \\
& A_{l}^{+} j_{l}(r)=-\left(\frac{\partial}{\partial r}-\frac{l}{r}\right) j_{l}(r)=-j_{l+1}(r) .
\end{aligned}
$$

Eqs. (B.6) and (B.7) can be combined into

$$
\boldsymbol{\sigma} \cdot \mathbf{p} F_{k m}(\mathbf{r})=i \kappa \operatorname{sgn}(k) F_{-k m}(\mathbf{r}),
$$

where $F_{k m}(\mathbf{r})=\chi_{k m}(\Omega) j_{l}(\kappa r)$ is the full bi-spinor. Then, if we construct the four-spinor as

$$
\Psi(\mathbf{r})=\left[\begin{array}{c}
F_{k m}(\mathbf{r}) \\
i \operatorname{sgn}(k) F_{-k m}(\mathbf{r})
\end{array}\right]
$$

it is clear that acting onto such a spinor by the Dimmock Hamiltonian (25) effectively transforms this Hamiltonian into a $2 \times 2 c$-number matrix

$$
H_{0} \rightarrow\left[\begin{array}{cc}
\left(\frac{E_{g}}{2}+\frac{\kappa^{2}}{2 m_{c}}\right) & P \kappa \\
P \kappa & -\left(\frac{E_{g}}{2}+\frac{\kappa^{2}}{2 m_{v}}\right)
\end{array}\right]
$$

so that the energy and $\kappa$ are related via

$$
\left[\frac{E_{g}}{2}+\frac{\kappa^{2}}{2 m_{c}}-E\right]\left[\frac{E_{g}}{2}+\frac{\kappa^{2}}{2 m_{v}}+E\right]+P^{2} \kappa^{2}=0
$$

For each specific energy this equation has four different solutions $\kappa$. Each pair $(\kappa,-\kappa)$ produces only a single linearly-independent solutions, so only two $\kappa$ 's out of the four are used to construct two linearly independent four-spinors. Each component of the linear combination of these two spinors has to vanish at the QD surface, which produces a homogeneous system of two equations with two unknowns. A secular equation has to be satisfied for this system to have non-trivial solution, thus resulting in the size-quantization condition. The explicit expression for the secular equation can be found in Ref. [22]. The solution of the Kang-Wise problem for the cylindrical symmetry is provided in Ref. [38]. 


\section{References}

[1] A. H. Ip, S. M. Thon, S. Hoogland, O. Voznyy, D. Zhitomirsky, R. Debnath, L. Levina, L. R. Rollny, G. H. Carey, A. Fischer, K. W. Kemp, I. J. Kramer, Z. Ning, A. J. Labelle, K. W. Chou, A. Amassian, E. H. Sargent, Nature Nanotech. 7 (2012) 577

[2] C. H. Chuang, P. R. Brown, V. Bulovic, M. G. Bawendi, Nature Mat. 13 (2014) 796.

[3] S. Ten Cate, C. S. Sandeep, Y. Liu, M. Law, S. Kinge, A. J. Houtepen, J. M. Schins, L. D. Siebbeles, Acc. Chem. Res. 48 (2015) 174.

[4] R. Schaller, V. Klimov, Phys. Rev. Lett. 92 (2004) 186601.

[5] V. I. Klimov, S. A. Ivanov, J. Nanda, M. Achermann, I. Bezel, J. A. McGuire, A. Piryatinski, Nature 447 (2007) 441.

[6] L. Kim, P. O. Anikeeva, S. A. Coe-Sullivan, J. S. Steckel, M. Bawendi, V. Bulovic, Nano Lett. 8 (2008) 4513.

[7] W. K. Bae, Y.-S. Park, J. Lim, D. Lee, L. A. Padilha, H. McDaniel, I. Robel, C. Lee, J. M. Pietryga, V. I. Klimov, Nature Comm. 4 (2013) 3661.

[8] L. Qu, X. Peng, J. Am. Chem. Soc. 124 (2002) 2049

[9] O. Chen, J. Zhao, V. P. Chauhan, J. Cui, C. Wong, D. K. Harris, H. Wei, H.-S. Han, D. Fukumura, R. K. Jain, M. G. Bawendi, Nature Mat. 12 (2013) 445.

[10] P. Y. Yu, M. Cardona, Fundamentals of Semiconductors: Physics and Materials Properties, 2nd ed., Springer-Verlag, Berlin, 2005.

[11] A. L. Efros, A. V. Rodina, Phys. Rev. B 47 (1993) 10005.

[12] H. Liu, P. Guyot-Sionnest, J. Phys. Chem. C 114 (2010) 14860

[13] R. C. Hilborn, Am. J. Phys. 50 (1982) 982.

[14] B. L. Wehrenberg, C. Wang, P. Guyot-Sionnest, J. Phys. Chem. B 106 (2002) 10634

[15] H. Haug, S. W. Koch, Quantum theory of the optical and electronic properties of semiconductors, fifth ed., World Scientific, 2009.

[16] E. Rosencher, B. Vinter, Optoelectronics, Cambridge University Press, Cambridge, 2002.

[17] V. B. Berestetskii, E. M. Lifshitz, L. P. Pitaevskii, Quantum Electrodynamics, volume 4, 2 ed., Pergamon, London, 1982.

[18] A. L. Efros, M. Rosen, Phys. Rev. B 58 (1998) 7120.

[19] L. C. Yan Voon, M. Willatzen, The kp Method: Electronic Properties of Semiconductors, Springer-Verlag, Berlin, 2009.

[20] G. Allan, C. Delerue, Phys. Rev. B 70 (2004) 245321.

[21] I. Moreels, K. Lambert, D. Smeets, D. De Muynck, T. Nollet, J. C. Martins, F. Vanhaecke, A. Vantomme, C. Delerue, G. Allan, Z. Hens, ACS Nano 3 (2009) 3023.

[22] I. Kang, F. W. Wise, J. Opt. Soc. Am. B 14 (1997) 1632

[23] L. D. Landau, E. M. Lifshitz, The Classical Theory of Fields, volume 2, 3 ed., Butterworth-Heinemann, Oxford, 1980.

[24] G. Bir, G. Pikus, Symmetry and Strain-Induced Effects in Semiconductors, Wiley, New York, 1974.

[25] Y. Aharonov, C. K. Au, Phys. Rev. A 20 (1979) 1553.

[26] E. O. Kane, J. Phys. Chem. Solids. 1 (1956) 82.

[27] C. Cercignani, E. Gabetta, Transport Phenomena and Kinetic Theory: Applications to Gases, Semiconductors, Photons, and Biological Systems, Modeling and Simulation in Science, Engineering and Technology, Birkhauser, Boston, 2007.

[28] G. E. Cragg, A. L. Efros, Nano Lett. 10 (2010) 313.

[29] N. Suzuki, K. Sawai, S. Adachi, J. Appl. Phys. 77 (1995) 1249.

[30] H. Kanazawa, S. Adachi, J. Appl. Phys. 83 (1998) 5997.

[31] J. O. Dimmock, G. B. Wright, Phys. Rev. 135 (1964) A821.

[32] J. O. Dimmock, in: D. L. Carter, R. T. Bates (Eds.), The Physics of Semimetals and Narrow-Gap Semiconductors, Pergamon, Oxford, 1971, p. 319.

[33] D. L. Mitchel, R. F. Wallis, Phys. Rev. 151 (1966) 581.

[34] T. B. Boykin, R. C. Bowen, G. Klimeck, Phys. Rev. B 63 (2001) 245314.

[35] B. A. Foreman, Phys. Rev. B 66 (2002) 165212.

[36] C. J. Pickard, F. Mauri, Phys. Rev. Lett. 91 (2003) 196401.

[37] P. Schwerdtfeger, C. van Wullen, J. R. Cheeseman, J. Chem. Phys. 137 (2012) 014107.

[38] S. V. Goupalov, Phys. Rev. B 84 (2011) 037303.

[39] A. R. Edmonds, Angular Momentum in Quantum Mechanics, Princeton University Press, Princeton, New Jersey, 1957.

[40] M. Abramowitz, I. A. Stegun, Handbook of Mathematical Functions, Dover, New York, 1965.

[41] W. R. Johnson, K. T. Cheng, M. H. Chen, in: P. Schwerdtfeger (Ed.), Relativistic Electronic Structure Theory, Part 2: Applications, Elsevier B.V., Netherlands, 2004, p. 120. 